\section{L'Actualité économique}

L'ACTUALITÉ ÉCONOMIQUE

Lyon, ville industrielle (Essai d'une géographie urbaine des techniques et des entreprises), par MICHEL LAFERRÈRE. Un vol., $6 \frac{1 / 2}{2}$ po. $\times$ 91/2, broché, XII et 546 pages, 32 figures, 4 planches hors-texte — PRESSES UNIVERSITAIRES DE FRANCE, Paris, 1960

\title{
Benoît Brouillette
}

Volume 37, numéro 1, avril-juin 1961

URI : https://id.erudit.org/iderudit/1001623ar

DOI : https://doi.org/10.7202/1001623ar

Aller au sommaire du numéro

Éditeur(s)

HEC Montréal

ISSN

0001-771X (imprimé)

1710-3991 (numérique)

Découvrir la revue

Citer ce compte rendu

Brouillette, B. (1961). Compte rendu de [Lyon, ville industrielle (Essai d'une géographie urbaine des techniques et des entreprises), par MICHEL

LAFERRÈRE. Un vol., $6 \frac{1}{2} 2$ po. x $91 \frac{1}{2}$, broché, XII et 546 pages, 32 figures,

4 planches hors-texte - PRESSES UNIVERSITAIRES DE FRANCE, Paris, 1960].

L'Actualité économique, 37(1), 193-195. https://doi.org/10.7202/1001623ar d'utilisation que vous pouvez consulter en ligne. 


\section{LES LIVRES}

Cette nouvelle édition représente, dans l'ensemble, une amélioration, un rajeunissement de l'édition antérieure qui ne datait que d'environ dix ans. On doit regretter qu'il n'existe pas de manuel équivalent écrit en langue française.

Le manuel de N. F. Keiser contraste violemment avec celui du professeur Cairncross. Le manuel de ce dernier contient peu de graphiques, peu de statistiques, sauf celles qui sont utiles, peu d'exemples arithmétiques (peut-être pas en assez grand nombre), pas de descriptions inutiles. L'ouvrage de Keiser, au contraire, renferme de tout en surabondance.

La division de l'ouvrage est plus ou moins arbitraire et nous parait désordonnée. Par exemple, le cinquième chapitre traite de la théorie des diverses formes de concurrence imparfaite; et plus loin, sans qu'on en comprenne la raison, l'auteur parle dans la section de la distribution du revenu, des diverses méthodes monopo listiques et du contrôle des monopoles.

On ne mentionne pas le concept de productivité marginale dans le chapitre de la distribution du revenu, alors qu'il est question, au début de l'ouvrage, de rendement décroissant et de coût marginal croissant. À la page 469, lauteur parle de l'utilité marginale du revenu sans qu'auparavant il ait défni l'utilité marginale. Il y a quelques bons chapitres, dont celui sur la formation des prix.

En voulant parler de tout, l'auteur ne réussit, à notre avis, qu'à donner une connaissance superficielle et confuse des notions essentielles de la science économique. On devrait s'attendre à ce que la multiplication des manuels améliore la présentation pédagogique des notions élémentaires d'économie politique. $\mathrm{Ce}$ nouveau manuel ne constitue pas un progrès dans ce sens, bien au contraire. Au moins aura-tril lavantage de faire connaître à quelques étudiants américains qui ne poursuivront pas davantage l'étude de l'économique, le visage de quelques économistes célèbres comme Malthus (p. 119), Smith (p. 28), Keynes (p. 224).

La comparaison de ces deux manuels destinés aux débutants montre la supé. riorité de celui du professeur Cairncross, manuel sobre, clair, bien écrit, sur celui de Keiser, ouvrage touffu et prolixe. Cette comparaison démontre aussi, sans doute, que deux qualités sont requises de celui qui veut écrire un bon manuel: une profonde culture économique et une riche expérience de l'enseignement.

Gilles DesRochers

Lyon, ville industrielle (Essai d'une géographie urbaine des techniques et des entreprises), par Michel LAFERRère. Un vol., $61 / 2$ po. $\times 91 / 2$, broché, XII et 546 pages, 32 figures, 4 planches hors-texte. - Presses Universitaires de France, Paris, 1960.

Les géographes américains ne sont pas les seuls à faire des études de géographie appliquée. L'un d'entre eux plaidait tout récemment la cause d'une géographie économique «plus humanistes en affirmant qu'il existe une géographie de l'entre. 


\section{L'ACTUALITE ÉCONOMIQUE}

prise $^{1}$. M. Michel Laferrère, professeur à l'Université de Lyon, n’a pas attendu cette injonction pour entreprendre l'étude de sa ville natale. Or, il l'a fait, non pas comme urbaniste, mais comme géographe qui s'est volontairement limité à deux caractères industriels de Lyon: la présence d'industries dans tous les quartiers de la ville, sauf dans le centre, et la variété des produits fabriqués.

Lyon est une ville surtout industrielle: 55 p.c. de ses 440,000 travailleurs dépendent directement de l'industrie. À la différence de ce que nous observons dans nos villes d'Amérique, on ne peut guère discerner de quartiers industriels individualisés. La croissance simultanée de Lyon et de ses industries explique le mélange des usines et ateliers dans les ensembles résidentiels; de sorte qu'il est difficile d'appliquer à Lyon une planification industrielle selon les règles classiques. Le plan ne peut que prévoir le maintien de certaines activités dans la ville, et l'expansion des usines à sa périphérie.

Le géographe ne se contente pas de cartographier et de décrire les industries; il propose une explication qui constitue l'essentiel de ses recherches. Examinons le contenu de l'ouvrage avant de le juger. Dans son introduction, l'auteur constate les avantages du site de Lyon et décrit les usines et ateliers dans le paysage urbain. Il en fait aussi une présentation statistique selon les dimensions des entreprises (prolifération des petites) et leur répartition spéciale d'après leurs effectifs.

Le corps principal du livre se partage en trois parties: la soierie lyonnaise (162 pages), la métallurgie (180 pages), la chimie ( 75 pages). La première, penseront certains, est en décadence. Cette opinion se révèle fausse: la soierie «demeure en France l'activité spécifique de Lyon,, affirme M. Laferrère, et il le prouve. Son texte clair, documenté, constitue la meilleure monographie qui ait été faite sur le sujet. L'existence d'activités métallurgiques à Lyon heurte également une idée «classique ; la métallurgie n'existe qu'en Lorraine ou dans le Nord, dit-on. Mais il s’agit, dans le cas de Lyon, non pas de métallurgie primaire, mais d'une industrie de transformation de matières venues de l'extérieur. C'est elle qui emploie le plus grand nombre d'ouvriers. Tandis que les textiles emploient 35,000 salariés, la métallurgie en utilise 88,280 , répartis dans 4,400 entreprises. Peu de grandes usines comptent plus de 500 personnes, mais 27 entreprises font travailler 45 p.c. des salariés de la métallurgie lyonnaise. Lyon compte dix usines dans le groupé de la construction électrique, cinq dans celui de l'automobile, quatre dans les domaines de la chaudronnerie et de la fabrication de wagons, deux dans le groupé de la mécanique générale; les autres se spécialisent dans les machines-outils, la robinetterie, les machines de précision, etc.

La chimie industrielle s'est établie à Lyon parce que cette ville est à la fois un carrefour de matières de base, un centre de transformation de demi-produits et enfin un milieu propice à l'utilisation de produits tels que les fibres synthétiques et les matières plastiques. Les salariés $(28,580)$ se partagent également entre là grande industrie chimique, la parachimie et les textiles artificiels. Cette jeune industrie, dont les effectifs ont augmenté de 7.5 p.c. en trente ans, s'est localisée

1. Robert B. McNee, -Towards a more humanistic economic geography: the geography of enterprise», Tijdschrift voor Economische en Sociale Geografie (Rotterdam), août 1960, pp. 201.205. 


\section{LES LIVRES}

dans les quartiers périphériques de Vaise et de Saint-Fons. L'usine Rhodiaceta de Vaise est la première fabrique française de nylon. À Saint-Fons, on trouve des firmes d'importance internationale, telles que Saint-Gobain, Ciba, Rhône. Poulenc et Air Liquide.

L'auteur, après avoir décrit les entreprises industrielles de sa ville, insiste sur le rôle que joue la recherche comme facteur du développement industriel de Lyon.

En l'absence d'autres facteurs, tels que les transports bon marché et l'abondance des capitaux, les Lyonnais ont fait preuve d'ingéniosité. Leur goût pour les voyages est aussi grand que leur capacité d'invention. Ils ont acquis un sens aigu de la concurrence. Cependant, remarque l'auteur, s'ils sont ingénieux, ses compatriotes sont parfois trop peu ingénieurs; bon commerçants, mais pas assez hommes d'affaires.

Le livre de M. Laferrère est remarquable non seulement par les renseignements qu'il contient, mais aussi par son analyse minutieuse des causes ayant fait de Lyon une ville industrielle aussi importante. Nous aurions beaucoup à faire pour étudier l'industrie montréalaise avec autant de profondeur. Ce livre peut servir de modèle; il montre clairement la voie à suivre. Les progrès industriels d'une ville ne se résument pas à des compilations statistiques tirées des documents officiels.

Benoit Brouillette

Syndicalisme et organisation professionnelle, Semaines Sociales du Canada (section française). Un vol., $61 \frac{12}{2}$ po. $\times 93 / 4$, broché, 242 pages. - Editions Bellarmin, 8100, boulevard SaintLaurent, Montréal 11, 1960. (\$3.50).

Il s'agit là d'un compte rendu des cours, «carrefours» et conférences de la XXXVII ${ }^{\mathrm{e}}$ session des Semaines Sociales du Canada tenue à Trois-Rivières en septembre 1960 sur le sujet énoncé plus haut.

Les Semaines Sociales du Canada avaient déjà étudié le syndicalisme, à Québec, en 1921 et l'organisation professionnelle à Trois-Rivières même, en 1936. Si cette année elles unissent les deux thèmes c'est, sans doute, comme le fait remarquer la lettre de Son Eminence le Secrétaire d'État du Vatican, afin de montrer qu'elles ne considèrent pas les deux structures comme des alternatives mais comme des compléments. En effet, continue la lettre du Vatican: *Le syndicalisme appelle une organisation professionnelle dans laquelle il vient s'intégrer; l'organisation professionnelle, à son tour, n'atteint pleinement son but que si elle s'appuie sur des unions librement constituées, où les différentes catégories sociales se sont groupées suivant leurs affinités et leurs intérêts propres.*

Il se peut que les Semaines Sociales paraissent revenir souvent sur les problèmes du travail. C'est sans doute parce que, d'une part, l'Eglise considère à bon droit ces problèmes comme fondamentaux dans une démocratie véritable et que, d’autre part, elle a une doctrine sociale apte à produire une démocratie véritablement sociale, susceptible de servir d'alternative entre la démocratie dite libérale (première expérience démocratique tentée au $\mathrm{XIX}^{\mathrm{e}}$ siècle et qui s'est soldée par 